\title{
Age-Specific Crisis Awareness Survey for Coronavirus Disease 2019 (COVID-19): How Much Do You Think You Should Pay for Hypothetical Prophylactic Vaccine and Therapeutic Drug for COVID-19?
}

Takashi Kawahara ( $\nabla$ takashi_tk2001@yahoo.co.jp)

Yokohama City University Medical Center https://orcid.org/0000-0002-7049-3379

Masahiro Yao

Yokohama City Uiniversity

Hiroji Uemura

Yokohama City University Medical Center

Research article

Keywords: generation, age, awareness, COVID-19, vaccine

Posted Date: August 28th, 2020

DOl: https://doi.org/10.21203/rs.3.rs-62958/v1

License: @ (i) This work is licensed under a Creative Commons Attribution 4.0 International License. Read Full License 


\section{Abstract}

BACKGROUND: The spread of coronavirus disease 2019 (COVID-19) has been a worldwide phenomenon with $2,063,161$ people infected and 136,938 deaths globally as of April 16,2020 . This study conducted a chronological survey of crisis awareness regarding COVID-19 within different age groups.

METHODS: An internet-based survey was performed of healthy Japanese adults to investigate the value of a hypothetical prophylactic vaccine and therapeutic drug for COVID-19 in each age group. The survey was conducted on Friday of each week commencing from February 14, 2020 to April 10, 2020. At certain times or events such as when the government released major announcements or when there was a rapid increase in the number of infected individuals, a similar survey was conducted on an additional 1,200 individuals per week.

RESULTS: A total of 12 surveys, including weekly surveys spanning over 9 weeks, were conducted, and a total of 19,113 samples from 12,254 individuals were obtained. The mean price for a hypothetical prophylactic vaccine was 2876.3 JPY (26.9 USD) at the first survey and was significantly increased to 3357.4 JPY (31.4 USD) for the most recent survey $(p<0.0001)$.

CONCLUSIONS: Though the percentage of those who will not pay were higher in young age group than in elderly age group, the percentage of those who will pay more than standard costs of influenza vaccine or treatment drugs were same between each aging group.

\section{Background}

The novel coronavirus infection, coronavirus disease 2019 (COVID-19) which first occurred in the Wuhan Province of China in 2019, has been spreading throughout the world since approximately February 2020 [1]. Although every country is trying to prevent the spread of COVID-19 through various isolation policies, the number of deaths continues to rise. As of April 16, 2020, the cumulative number of COVID-19 patients worldwide was 2,063,161, along with 136,938 deaths confirmed [2]. The risk of deaths is particularly high in individuals with underlying diseases and the elderly, while younger individuals often exhibit mild or asymptomatic disease with overall low mortality rates. Thus, the differences in crisis awareness in relevance to the disease between age groups have been an issue [3]. We therefore conducted a prospective study, starting in February 14, 2020, when only a small number of COVID-19 cases were noted in Japan, to determine how age range and the spread of infection affected crisis awareness.

\section{Materials \& Methods}

\section{Screening survey}

From February 14, 2020, a questionnaire type survey form was administered via iBRIDGE's Freeasy, an internet survey firm (Freeasy; iBRID GE Corporation, Tokyo, Japan). Healthy individuals were randomly selected each week from 4.5 million panels at Freeasy based on age and gender parameters. Two questionnaires were administered to a total of 2,400 individuals on a weekly basis (weeks 1-4) who were split into 12 groups comprising of males and females in 6 different age groups (i.e. 20s-60s, 70 years or older), because it was initially expected that the spread of COVID-19 would end early. Subsequently, a total of 1,200 individuals were administered weekly as it was expected that there would be a global spread and prolongation to the end of the spread of infections. In addition, 1,200 additional individuals were administered whenever the World Health Organization (WHO) or the Japanese 
government issued announcements or there were major changes in infection status. Since the questionnaire was, for the most part, completed within one day, it was considered that there would be no overlap in the questionnaire period. In addition to sex and age, information on prefecture/city of residence, type of residential facility, annual household income, marriage status, and the presence or absence of children were obtained together. Monitoring and evaluation were consented during the online survey at the time of answering the questionnaire, while the Institutional Review Board (IRB) at Yokohama City University Medical Center (Yokohama, Japan) approved this study (IRB No. B200300041).

\section{Questionnaire}

To investigate crisis awareness regarding COVID-19 in healthy Japanese adults, the values assigned to a hypothetical COVID-19 prophylactic vaccine and hypothetical COVID-19 treatment drugs were compared to the price paid for the domestic influenza vaccine and other influenza drugs in Japan. Japan has a universal insurance system that is different from those in most of other countries. Under this system, almost all Japanese nationals have access to the uniform treatment regardless of the employment status or income. Conversely, since the proportion of out-of-pocket payments varies based on income and insurance type, there is a difference in the amount paid by an individual who receives the same treatment (about $70-90 \%$ of medical costs are covered by insurance). The influenza vaccine is administered annually to approximately $50 \%$ of the population or 60 million of Japanese nationals, and the average out-of-pocket amount for each individual in Japan is about 3,600 JPY (33.6 USD). Moreover, crisis awareness regarding COVID-19 in healthy individuals was investigated in the form of asking individuals what they thought the expected payment should be for both a hypothetical prophylactic vaccine and hypothetical therapeutic drugs specific for COVID-19, assuming that out-of-pocket expenses including that of medical care and drugs would be around 3,000-6,000 JPY (28.0-56.1 USD) (although there would be a range in the cost of drugs for treatment). A crisis awareness survey consisted of two simple questions, "How much would you pay for a vaccine against COVID-19?" and "How much would you pay for treatment for COVID-19?" [Supplementary Material].

The following parameters were compared as indices of crisis awareness: number of deaths in Japan and around the world, and the cost of a hypothetical prophylactic vaccine and treatment drugs. The proportion of individuals who were willing or unwilling to pay more than the price of the influenza vaccine and drugs was also evaluated. Additional surveys were conducted to determine the impact of major announcements from the Japanese government, WHO, or similar bodies, as well as the timing of such events.

\section{Comparison with the number of patients}

The numbers of infected individuals and patient deaths in Japan and worldwide were based on those reported by the Japanese Ministry of Health, Labour and Welfare and data from the Johns Hopkins Coronavirus Resource Center, respectively [4].

\section{Statistical analyses}

The participants' characteristics and scores were analyzed by Mann-Whitney $U$ test using the Graph Pad Prism software program (Graph Pad Software, La Jolla, CA, USA). P values that were $<0.05$ were considered to be statistically significant.

\section{Results}


The study was conducted on 19,113 samples (12,254 individuals) with a total of 12 surveys in the timeframe between February 14, 2020 and April 12, 2020. These included weekly surveys that were administered every Friday, three additional surveys, and others administered during events such as major announcements from the government and when there was a drastic rise in the number of infected individuals [Fig. 1, Table 1, supplementary Table 1]. On February 14, 2020 (Wk1), the number of individuals in Japan who were positive for COVID-19 test was only 33 , and 63,851 (95.4\%) out of 66,900 positive cases in the world were from China. On April 10, 2020 (Wk9), there was an explosive increase in the number of positive cases in Japan (5,246 individuals) or globally (1.7 million) [Fig. 1].

Table 1

Background of the study samples

\begin{tabular}{|ll|}
\hline Variables & median (mean+/-SD) or number (\%) \\
\hline Number of samples (cases) & 19,113 samples (12,254 cases) \\
\hline Age (yrs.) & $49(49.5+/-16.8)$ \\
\hline Gender & \\
\hline Male & $9510(49.8 \%)$ \\
\hline Female & $9603(50.2 \%)$ \\
\hline Married & $11428(59.8 \%)$ \\
\hline Child & $9732(50.9 \%)$ \\
\hline Household Income (/year) & \\
\hline$-2,000,000 J P Y$ & $3,177(8.7 \%)$ \\
\hline 2,000,001-4,000,000JPY & $5,235(12.7 \%)$ \\
\hline $4,000,001-6,000,000 J P Y$ & $4,494(13.1 \%)$ \\
\hline $6,000,001-8,000,000 J P Y$ & $2,613(7.0 \%)$ \\
\hline $8,000,001-10,000,000 J P Y$ & $2,391(4.3 \%)$ \\
\hline 10,000,001-JPY & $1,203(2.9 \%)$ \\
\hline Working Pattern & $3,267(17.1 \%)$ \\
\hline Housewife & $3,556(18.6 \%)$ \\
\hline Employee & $2,277(27.6 \%)$ \\
\hline Part-time job & $982(5.1 \%)$ \\
\hline Temporary job & $3,560(18.6 \%)$ \\
\hline No-occupation & \\
\hline Others & \\
\hline
\end{tabular}

In this study, healthy individuals answered the amounts they would consider paying for a hypothetical prophylactic vaccine and therapeutic drugs for COVID-19. Results showed that, as the number of COVID-19 infections 
increased, the prices allocated for the hypothetical vaccine and drugs increased till Wk8.2 [Figs. 2 and 3]. However, when Japanese government declared a state of emergency at Wk8.4, the prices allocated for the hypothetical vaccine and therapeutic drugs were decreased [Figs. 2 and 3]. Prices for the hypothetical prophylactic vaccine for COVID-19 were significantly increased from 2876.3 JPY (26.9 USD) at Wk1 to 3357.4 JPY (31.4 USD) at WK9 (p< 0.001). A temporary decrease in the hypothetical prices was noted on March 20-22, 2020 (Wk6) during a long weekend when an increase in the number of infected individuals was not reported in Japan. An increase in the hypothetical price was also seen upon the report of the death of a famous Japanese comedian "Ken Shimura" due to COVID-19 on March 30, 2020 at Wk7.3 [Fig. 2].

Across the different age groups, the prices for the hypothetical prophylactic vaccine for COVID-19 have been significantly higher in the elderly group than in the younger age group at Wk5 when the number of infected individuals domestically exceeded comparing to the previous several weeks [Fig. 4a]. The percentage of those who were not willing to pay for prevention was higher in the young group compared to the elderly group, but the percentage of those who were willing to pay for prevention more than prophylaxis influenza vaccine showed almost same between each age group. As when the number of positive COVID-19 case has been increases in Japan, the percentage of those who were willing or unwilling to pay for prevention was increased or decreased, respectively [Fig. 4b and 4c] For the treatment, the elderly group was willing to pay more than younger group but there were no significantly differences [Fig. 4d]. The percentages of those who were not willing to pay for prevention were higher in the young group and lower in the elderly group. The percentage of all participants who were not willing to pay for the treatment was decreased when the COVID-19 was widespread [Fig. 4e]. The percentage of those who were willing to pay for the treatment was similar in the all age groups during all phases but was gradually increased as COVID-19 was widespread [Fig. 4f and 4g].

In further sub-group analysis, the percentage of females who were willing to pay more than standard influenza vaccine for prevention and the amount were significantly higher at Wk9, compared with those in males [Fig. 5a]. At Wk8.4 Japanese government declared state of emergency in 7 prefectures. When compared between these 7 prefectures (area) and the other area (non-area), there were no differences before state of emergency, but after the state of emergency, those in the area were willing to pay more than those in the non-area [Fig. 5b]. In terms of household income, in an early era, higher household income groups were willing to pay higher amounts, but the differences were then diminished [Fig. 5c]. In addition, those who were married and had child(ren) were more likely willing to pay for prevention at all phases [Fig. $5 \mathrm{~d}$ and $5 \mathrm{e}]$.

\section{Discussion}

This study was a large-scale survey from 19,113 samples and 12,254 individuals over 9 weeks. Although low levels of crisis awareness in younger age groups have been reported in media coverage and other small-scale awareness surveys, this was the first objective prospective survey to investigate this topic. We found that the increasing spread of COVID-19 throughout Japan more strongly correlated with an increased potentially awareness of prevention and treatment for COVID-19 than the spread of COVID-19 worldwide. Additionally, a deeper awareness of treatment further increased with the reported death of a famous Japanese celebrity comedian on March 30 (Wk7.3). Reports on the rise of individuals who were positive for COVID-19 test from the Diamond Princess cruise line from February 5 to March 1 (Wk1-3) temporarily resulted in increased crisis awareness. In these periods, there were no significant differences in crisis awareness and the cost of hypothetical vaccine and drugs showed almost flat trend in not only the younger age group but also the elderly. 
In Japan, as there was no significant rise in the number of infected individuals in these survey time, there seemed to be a sense of security in individuals. This more individuals, combined with the fact that it was also cherry blossom viewing season, more individuals ventured outside during the long weekend from March 20-22. In this study, the prices allocated for the hypothetical prophylactic vaccine and other therapeutic drugs were also reduced on March 20 (Wk6), and the survey seemed capable of reflecting crisis awareness in Japanese individuals. Moreover, an additional survey conducted at the time of the reported death of a famous comedian in Japan showed a sharp rise in awareness.

Differences in crisis awareness between the younger age group and the elderly were believed to result from agerelated differences, such as the increase in severity of symptoms and higher mortality rates related to age. Mortality rates associated with COVID-19 increased with age, with $0.2 \%$ deaths in those in the $20 \mathrm{~s}$ or $30 \mathrm{~s}, 0.4 \%$ in those in the $40 \mathrm{~s}, 1.3 \%$ in those in the $50 \mathrm{~s}, 3.6 \%$ in those in the $60 \mathrm{~s}, 8.0 \%$ in those in the $70 \mathrm{~s}$, and $14.8 \%$ in those 80 years or older [5]. For this reason, the assumption is that the younger age group is less aware of the crisis and consequently has lower compliance rates to governmental directives, such as bans on going outside. However, the percentage of cases who were not willing to pay for the prevention and treatment was reduced correlating with increases in the number of COVID-19 positivity in Japan. The proportions of participants who were willing to pay for the treatment more than the standard price for the influenza drugs in any phase were similar in all age groups and were increased with widespread of COVID-19.

In this study, crisis awareness was evaluated based on a comparison of the cost for prophylactic and treatments with that for influenza. There are currently no questionnaires that assess awareness of treatment and sense of crisis that are validated in the Japanese language. It is currently unknown whether questionnaires would be suitable for this infectious disease. In this regard, the idea was that performing a comparison to those of influenza, an infectious disease for which prophylactic vaccination and treatment were provided in about $50 \%$ of individuals in Japan would be convenient. Japan ranks 16th in the Organisation for Economic Co-operation and Development members in influenza vaccination rate in those aged 65 , with the Republic of Korea ranking highest at $83 \%$, followed by Australia, the United Kingdom, and the United States [7]. Specifically, in 2020, the percentage of people vaccinated for influenza was $58.5 \%$ in the elderly, $28.6 \%$ in adults, and $59.2 \%$ in children [6]. It is also considered easy to assess the number of infected individuals since there has been a constant frequency of 200-300 million individuals in Japan each year [8]. Thus, we used this influenza comparing questionnaire due to the popular disease in Japan. Conversely, the mean cost of a hypothetical prophylactic vaccine and other hypothetical prophylactic drugs were 2876.3 JPY (26.9 USD) and 3357.4 JPY (31.4 USD) at Wk9, respectively, and considerably lower in situations where the government declared an emergency comparing to seasonal influenza.

This study evaluated the potentially demand for medical care in healthy individuals. In particular, in patients with severe symptoms of COVID-19, the medical load is higher with measures in place such as ventilator management and this higher load can lead to a higher burden on medical workers. Consequently, it may also be the cause of medical disruption which can lead to higher mortality rates. Since Japan, in particular, still has lower morbidity and mortality rather compared to those of Western countries at the time of this study, the belief is that raising awareness in individuals without symptoms will be necessary. This study might be clue to evaluate the potential crisis awareness for COVID-19.

This study had the following limitations: the validity of the questionnaire itself, lack of detailed evaluations including the ability to perform evaluations over time despite issues with awareness remaining. But this simple survey, could evaluate the results regardless of final educational attainment. 
The study revealed a change in crisis awareness in all age groups, which was associated with an increasing awareness of infected individuals. Moreover, crisis awareness was lower in the younger age group than in the elderly, whereas crisis awareness in the middle-aged group fluctuated between high and low levels. Meanwhile, females, married persons, and those with child(ren) showed higher crisis awareness.

\section{Abbreviations}

COVID-19: coronavirus disease 2019

WHO: World Health Organization

\section{Declarations}

\section{Ethics approval and consent to participate}

The institutional review board of Yokohama City University Medical Center (Yokohama, Japan) approved this study (IRB No: B200300041).

\section{Consent for publication}

The institutional review board of Yokohama City University Medical Center (Yokohama, Japan) approved this study (IRB No: B200300041).

\section{Availability of data and material}

The raw data to create tables and figures are available as a supplementary file.

\section{Competing interests}

We declare no conflicts of interests of all authors.

\section{Funding}

None

\section{Authors' contributions}

TK wrote the manuscript. TK, YM, HU performed the experiment.

\section{Acknowledgements}

Native English language check was performed by Japan Medical Communication.

\section{References}

1. Kamel Boulos MN, Geraghty EM: Geographical tracking and mapping of coronavirus disease COVID-19/severe acute respiratory syndrome coronavirus 2 (SARS-CoV-2) epidemic and associated events around the world: how 21 st century GIS technologies are supporting the global fight against outbreaks and epidemics. Int $J$ Health Geogr 2020, 19(1):8. 
2. (JHU) CaJHU: Coronavirus 2019-nCoV Global Cases by Johns Hopkins CSSE.

https://gisanddatamapsarcgiscom/apps/opsdashboard/indexhtml\#/bda7594740fd40299423467b48e9ecf6.

3. Brodin P: Why is COVID-19 so mild in children?Acta Paediatr 2020.

4. Ministry of Health L, and Welfare: About Coronavirus Disease 2019 (COVID-19).

https://wwwmh/wgojp/stf/seisakunitsuite/bunya/newpage_00032html.

5. Wu Z, McGoogan JM: Characteristics of and Important Lessons From the Coronavirus Disease 2019 (COVID19) Outbreak in China: Summary of a Report of 72314 Cases From the Chinese Center for Disease Control and Prevention. JAMA 2020.

6. Hiroaki Nobuhara YW, Yoshihiko Miura: Trends for Influenza vaccinization in Japan. Journal of Japanese Society of Public Health 2014, 61(7):354-359.

7. (2019) O: Health at a Glance 2019: OECD Indicators. OECD Publishing, 2019.

8. Ministry of Health $L$, and Welfare: Goverment release.

https://wwwmh/wgojp/stf/seisakunitsuite/bunya/kenkou_iryou/kenkou/kekkaku-

kansenshou01/houdouhtm/2019.

9. YouGOV: You Gov international COVID-19. https://yougovcouk/topics/international/articlesreports/2020/03/17/YouGov-international-COVID-19-tracker.

\section{Figures}

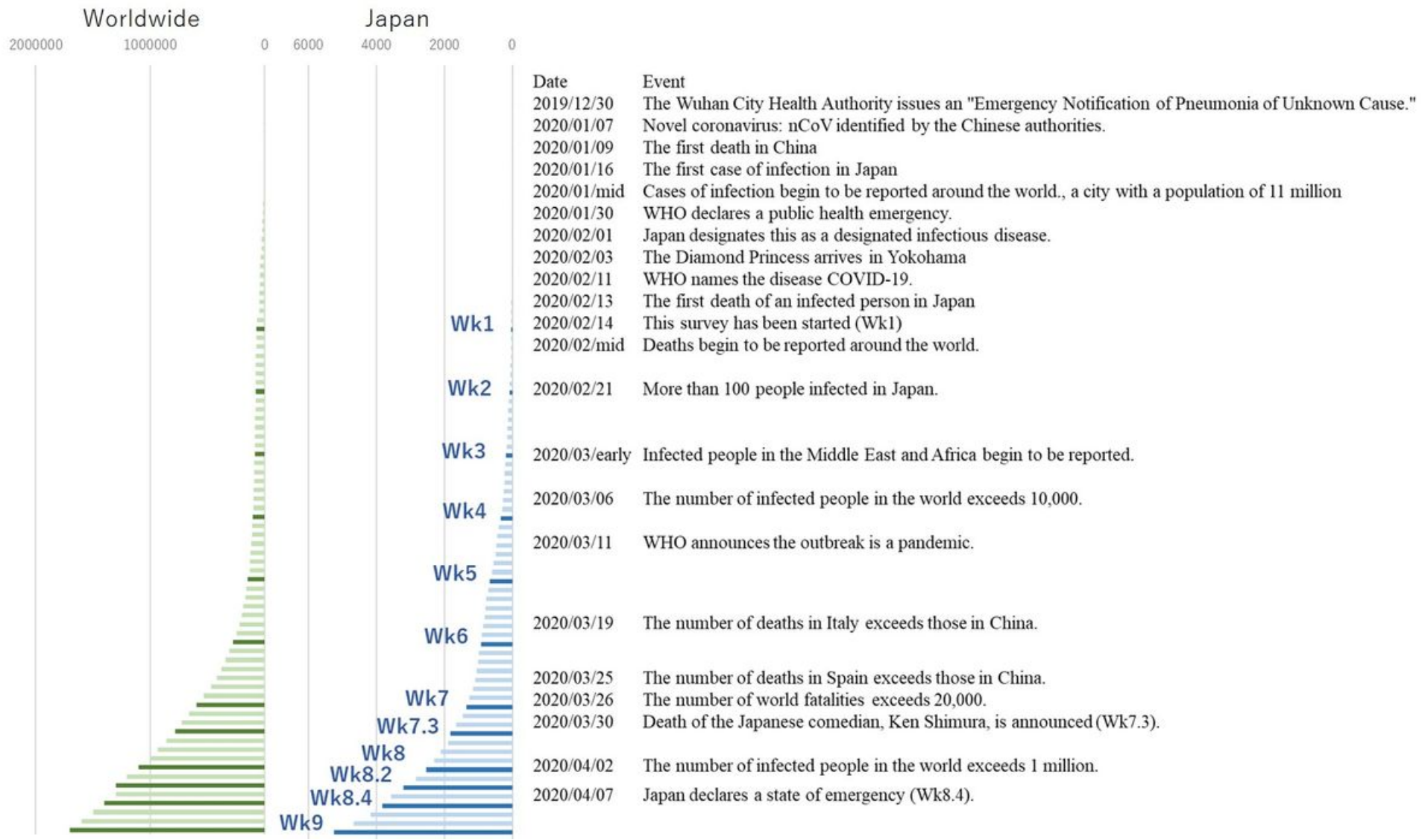

Figure 1

History of COVID-19 and number of patients.. Green: Worldwide cases, Blue: Japanese cases. 


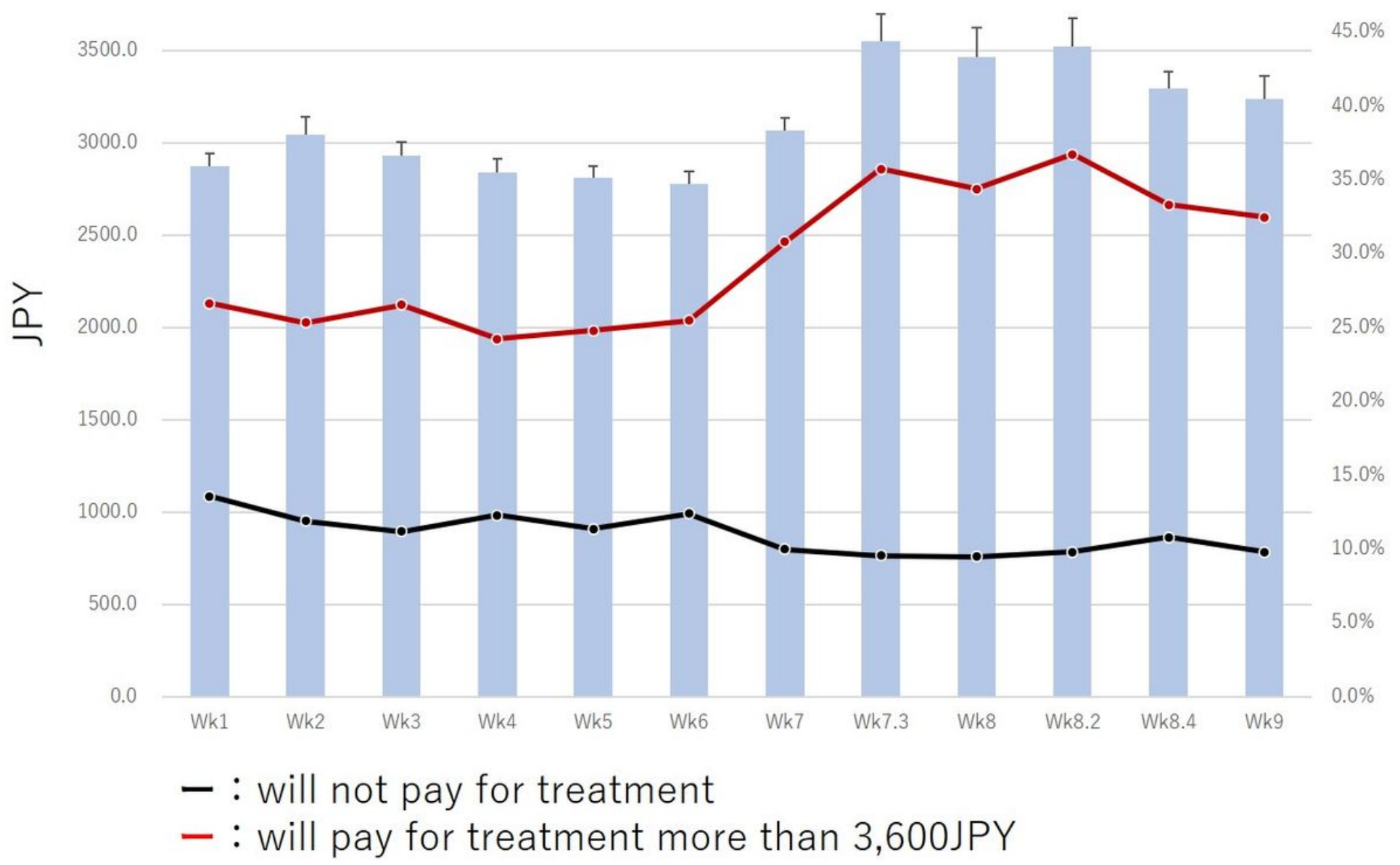

Figure 2

Transition of hypothetical COVID-19 vaccine cost (Row). Percentages of cases who would pay more than 3,600 JPY (red line) and who would not pay (black line) for this vaccine are shown. 


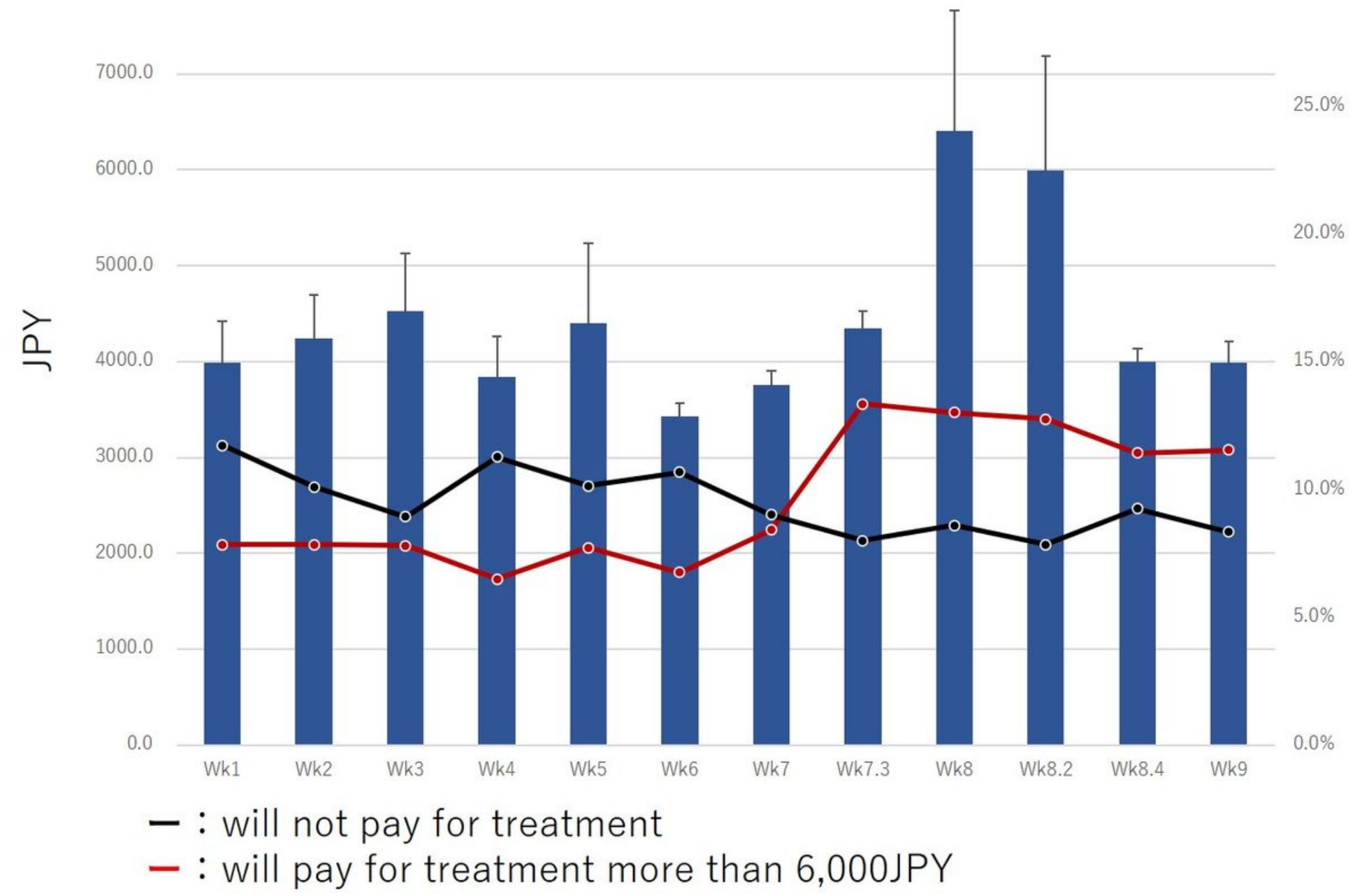

Figure 3

Transition of hypothetical COVID-19 treatment cost (Row). Percentages of cases who would pay more than 6,000 JPY (red line) and who would not pay (black line) for this treatment are shown. 


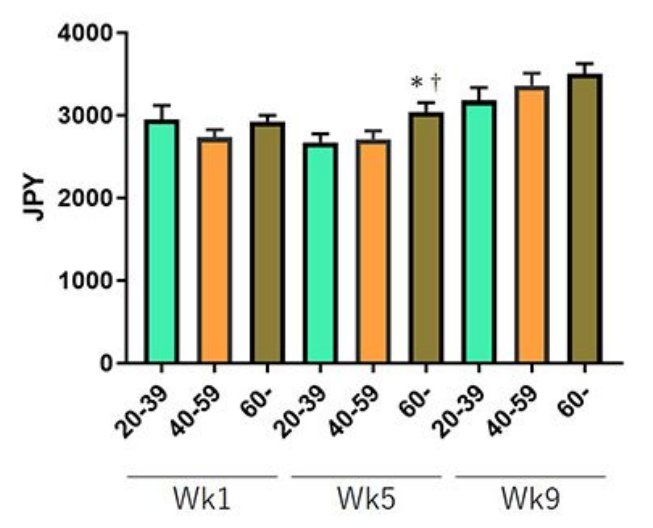

${ }^{*},{ }^{\dagger}: \mathrm{p}<0.05$ (vs Wk5 20-39, Wk5 40-59)
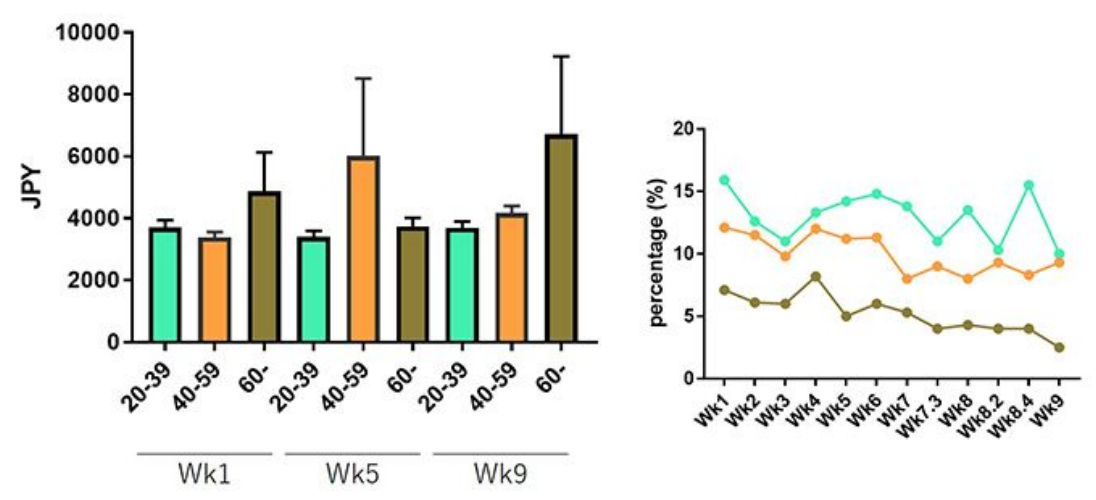
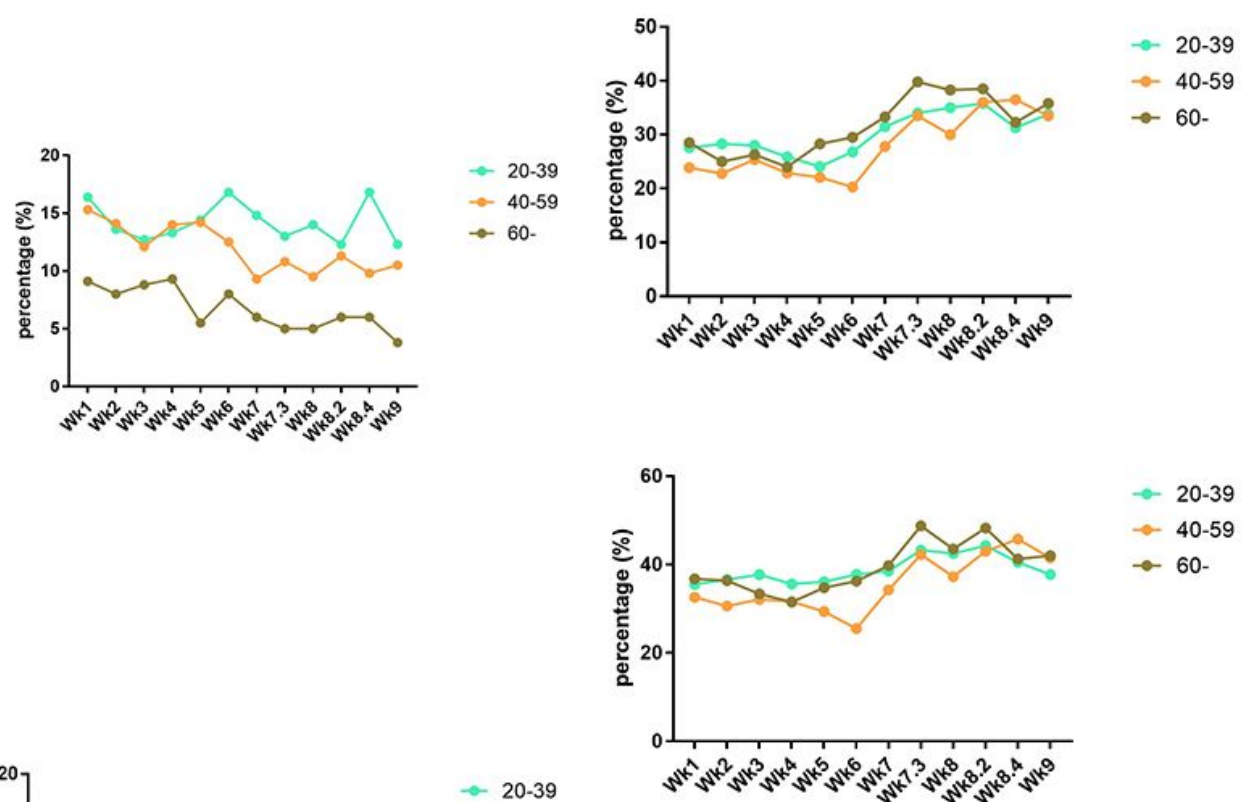

$\rightarrow \quad$ 20-39

$\rightarrow 60$

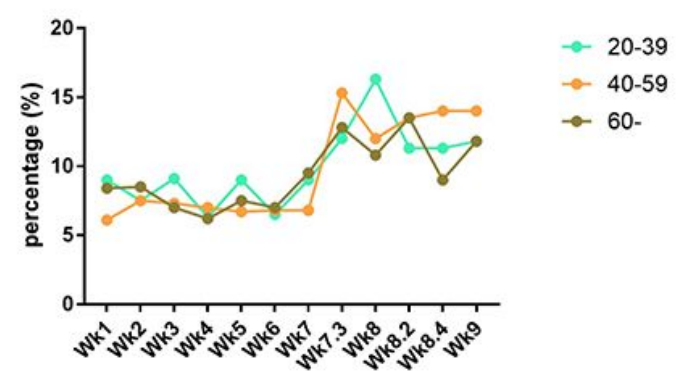

\section{Figure 4}

The differences for prevention and treatment of COVID-19 among age groups. a) Hypothetical prevention cost at Wk1, Wk5, and Wk9. b) Percentage of cases who were not willing to pay for a vaccine. c) Percentage of cases who were willing to pay more than 3,600 JPY for a vaccine. d) Hypothetical treatment cost at Wk1, Wk5, and Wk9. e) Percentage of cases who were not willing to pay for the treatment. $\mathrm{f}$ ) Percentage of cases who were willing to pay more than 3,600 JPY for the treatment. d) Percentage of cases who were willing to pay more than 6,000 JPY for the treatment. 

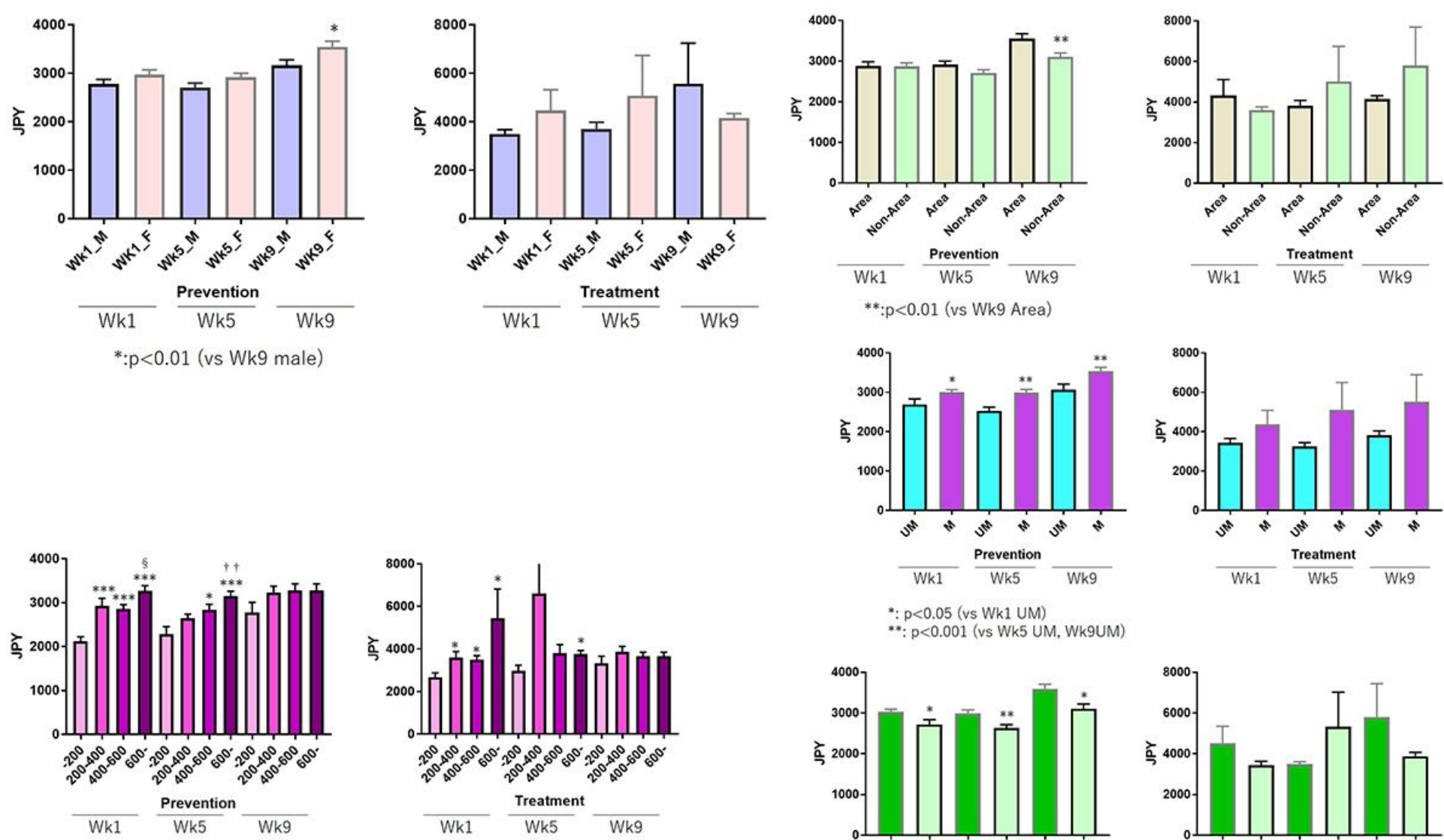

*: $p<0.05$ (vs Wk1 UM)

$* *: p<0.001$ (vs Wk5 UM, Wk9UM)
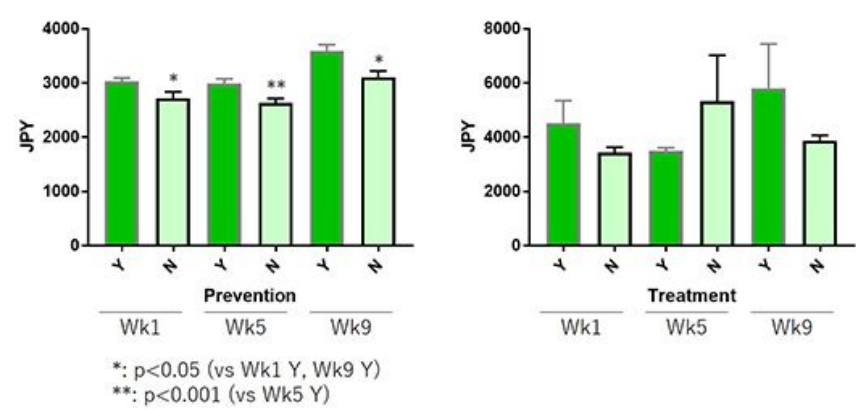

\section{Figure 5}

The differences in a) gender, b) area, c) annual household income, d) marriage status, and e) presence of child(ren) for prevention and treatment of COVID-19.

\section{Supplementary Files}

This is a list of supplementary files associated with this preprint. Click to download.

- AdditionalFiles.docx

- stable1R0.docx

- ncoronaENGquestionnaireHM.docx 